\title{
Aprendiendo las Partes del Átomo de Hidrógeno con un videojuego
}

\author{
doi: 10.33264/rpa.202101-11 \\ Luis Pérez, Mauricio Saenz Correa \\ Escuela de Informática UNIACC \\ Facultad de Administración UNIACC
}

\section{Resumen}

Hoy en día, los niños destinan a los videojuegos gran tiempo de sus vidas. Bajo este escenario, es que se midió el impacto en el aprendizaje de la estructura básica de un átomo de hidrógeno, que puede tener la utilización de un videojuego por cinco días para nueve niños. Inicialmente se trabajó con 37 niños chilenos de 8 vo básico para conocer su uso e interés en los videojuegos, por medio de un cuestionario con preguntas basadas en escala Likert. Luego se aplicó un cuestionario sencillo de conocimientos previos, en que debían identificar las partes del átomo de hidrógeno, denotando el poco conocimiento de este. Finalmente, se trabajó con dos grupos de estudiantes, uno que estudió los contenidos con una ficha tradicional de contenido (18 alumnos) y otro que además trabajó con el videojuego (10 alumnos). Al finalizar, se aplicó un nuevo instrumento para la evaluación de los contenidos, presentado resultados positivos, mostrando un mejor desempeño para el grupo de nueve niños respecto a su aprendizaje de las partes del átomo de hidrógeno. Esto deja en evidencia un potencial que tiene el uso de esta tecnología para el proceso educativo.

Palabras clave: Videojuego educativo, gamificación, átomo de hidrógeno, innovación educativa, bosón de Higgs

\section{Abstract}

Today, children spend a great time of their lives playing video games. Under this scenario, the impact on learning the basic structure of a hydrogen atom was measured, which can have the use of a video game for five days for ten children. Initially, we worked with 37 8th grade Chilean children to learn about their use and interest in videogames, through a questionnaire with questions based on the Likert scale. Then a simple questionnaire of previous knowledge was applied, in which they had to identify the parts of the hydrogen atom, denoting the little knowledge of this. Finally, we worked with two groups of students, one who studied the content with a traditional content card (18 students) and the other who also worked with the video game ( 9 students). In the end, a new instrument for the evaluation of the contents was applied, presenting positive results, showing better performance for the group of nine children regarding their learning of the parts of the hydrogen atom. This reveals the potential that the use of this technology has for the educational process.

Keywords: Serious videogame, gamification, hydrogen atom, educational innovation, Higgs boson 


\section{Introducción}

El objetivo del presente trabajo ha sido medir cuantitativamente el impacto de un videojuego en relación con un tema específico del ámbito del conocimiento científico, en particular la estructura del átomo de hidrógeno considerando las subpartículas atómicas (Ministerio de Educación, 2018), en alumnos de 8 vo básico de la educación chilena. Es decir, tanto en los modelos atómicos de Thomson, Rutherford y Bohr, los estudiantes conocen los diferentes planteamientos, y logran diferenciar el núcleo atómico, que es donde ubican los protones y neutrones, y las órbitas exteriores donde se encuentran los electrones. Junto con esto, con el videojuego se pretende presentar que tanto los protones como los neutrones estaban formados por partículas más pequeñas llamadas quark arriba y quark abajo, las que se mantienen unidas gracias a la interacción de los gluones. Y que los electrones por otro lado eran realmente partículas sin componentes más pequeños. También se les menciona el Bosón de Higgs como el causante de dar masa a las partículas. Todo esto último de acuerdo con la teoría del modelo estándar de la física de partículas, desarrollada (y en desarrollo) durante los últimos 60 años. Cabe hacer notar que hace un siglo, los físicos como Schrödinger, Heisenberg, y Dirac entre otros iniciaron lo que se conoce desde entonces como la Física Cuántica.

Para medir los conocimientos de estos temas se aplicaron instrumentos ex ante y ex post en relación con el momento en que fue conocida esta materia por parte de los estudiantes, donde algunos de ellos se enfrentaron a jugar con un videojuego desarrollado para estos fines en comparación con otros que avanzaron en su conocimiento por medio del método tradicional.

\section{Situación Actual}

La "ludificación" se define como el "uso de técnicas, elementos y dinámicas propias de los juegos y el ocio en actividades no recreativas con el fin de potenciar la motivación, así como de reforzar la conducta para solucionar un problema, mejorar la productividad, obtener un objetivo, activar el aprendizaje y evaluar a individuos concretos" (Galán, 2019), la ludificación es más conocida por el anglicismo gamificación, del inglés gamification, término acuñado el año 2002 por el Programador Británico de juegos Nick Pelling (Yip, 2015).

En particular, la gamificación de un proceso de aprendizaje debe ser desarrollado de una manera estructurada y no por el simple hecho de forzar el contar con algún elemento de videojuego. En general, cuando esto sucede, la experiencia no es exitosa (Mora, Riera, González \& Arnedo-Moreno, 2015). En el mundo moderno, los intereses de los alumnos han cambiado, por lo que los profesores necesitan explorar nuevos recursos en sus clases para aumentar la motivación y el compromiso de sus alumnos. En el trabajo de Ortiz-Colón, Jordán y Agredal (2018) se presenta que los procesos de gamificación en educación generan en los alumnos importantes beneficios, tales como la motivación, la inmersión, el compromiso y la socialización, que hacen a la actividad educativa más motivante y estimulante para los alumnos. 
El utilizar videojuegos para el aprendizaje permite llevar experiencias de la vida cotidiana a la educación de los alumnos, aumentar la motivación y el compromiso con el aprendizaje, y acercarse a los estilos actuales de aprendizaje de estos (James, Beaton, Csete \& Vogel, 2003). Muchos autores han analizado el impacto de los videojuegos en el desarrollo de habilidades de resolución de problemas. Algunos de ellos creen que pueden promover un aprendizaje de alto orden, como incrementar diálogos significativos entre los alumnos (Garrido, 2013; Lim, Nonis, Hedberg, 2006), o mejorar su movilidad y orientación (Sánchez, Saenz, Ripoll, Garrido, 2010). Así mismo, estudios describen los efectos positivos de los videojuegos en habilidades sociales (González, 2014; Pallegrini, Blatchford, Kentaro, 2004); contenidos de historia (Evaristo, Navarro, Vega, Nakano, 2016) y aprendizaje de la ciencia (Lim, Nonis, Hedberg, 2006; Sánchez, Saenz, 2008; Sánchez, Saenz, Salinas, 2009).

Un videojuego de código abierto, de nombre Lincity, ha servido para la implementación de una experiencia de una secuencia didáctica para desarrollar conceptos de energía dentro del espacio curricular para la introducción de la física. Para los investigadores Farías, Fornasier, Moro y Morcela (2016), el uso de estos videojuegos permite evidenciar la práctica, tomar decisiones y analizar sus consecuencias, siendo por tanto una puerta de entrada importante para la revisión y aprendizaje de determinados contenidos. Para el éxito de estas experiencias resulta fundamental la motivación y es ahí donde entra el uso de videojuegos.

"Dynamo, ciudad de la física" (2018), es un videojuego que busca explicar las nociones básicas asociadas al manejo de cifras, vectores y modelos de conversión de unidades. Este videojuego utiliza simulaciones del mundo real como un gimnasio y un concierto, para el manejo de transformación de unidades y el manejo de voltajes dentro de diversos equipos. En un ambiente móvil se presenta el videojuego "Protectores de la Ciencia: Método científico" (2015) que busca explicar de una manera entretenida y didáctica los pasos asociados a la investigación científica. Gracias a estos será posible ir resolviendo misiones, en que a medida que se avanza en estas, el nivel de complejidad irá creciendo.

Se pueden ocupar videojuegos para resolver problemas complejos como pueden ser los relacionados a la física cuántica. En el trabajo de Sorensen et al. (2016) muestran un videojuego de nombre Quantum Moves, que es proporcionado en línea para jugar utilizando operaciones que hoy en día se realizan en computación cuántica de manera sencilla. Otros videojuegos que han implementado se presentan dentro del sitio Science At Home (2016) perteneciente a la Universidad Aarhus de Dinamarca, donde presentan diversos títulos en que han logrado trasladar los cálculos de la física cuántica hacia los videojuegos. Una desventaja de estos videojuegos es que se encuentran en idioma inglés, lo que lo aleja de la posibilidad de un uso masivo en un país de habla hispana como Chile. Esto genera una gran oportunidad para proyectos como el expuesto en este artículo. 


\section{El Videojuego}

\section{Objetivo}

El objetivo del videojuego es lograr una mejora en el aprendizaje del reconocimiento de las partes del átomo de hidrógeno, teniendo claridad de los nombres de los diferentes elementos, además de su representación.

\section{Diseño}

El videojuego consistió en tres niveles con tres etapas cada uno, teniendo además un tutorial en donde se enseñaba el mecanismo del juego y además contenía una ayuda teórica que indicaba cual era la misión para conseguir, que era "armar" un átomo de hidrógeno.

En los tres niveles el jugador debía esquivar a los múltiples Bosones de Higgs, ya que se les indicaba que estos agregaban la masa a las partículas y por lo tanto había un contacto crítico que no debían superar, sino perderían el juego. En el primer nivel, el jugador controlaba al personaje quark up, el cual tenía como misión capturar tres gluones para pasar al siguiente nivel, las tres etapas del nivel iban aumentando en dificultad. En el segundo nivel, el jugador era un gluón que debía capturar a los tres quarks (dos quarks up y un quark down) para formar el protón, y al igual que en el nivel anterior debía sortear con éxito tres etapas. En el tercer y último nivel, el jugador era el protón y debía capturar al electrón para formar el átomo de hidrógeno.

Para darle un contexto más inmersivo al videojuego, los diferentes niveles fueron ambientados con una música electrónica futurista.

\section{Interfaces}

En la etapa tutorial, se presenta un esquema de las partes básicas del átomo hidrógeno, por medio de un esquema básico, pudiendo acercar a los alumnos esta materia. Se deja claro que este átomo será el protagonista del juego, entregando además información de cuál es la finalidad del juego. Como se ve en la figura 1, se muestra una representación del átomo que ya conocen en sus clases de ciencias naturales, pero con el agregado de que un protón se forma por la interacción de tres Quarks con los respectivos Gluones. 
Figura 1. Interfaz Tutorial: Átomo de Hidrógeno.

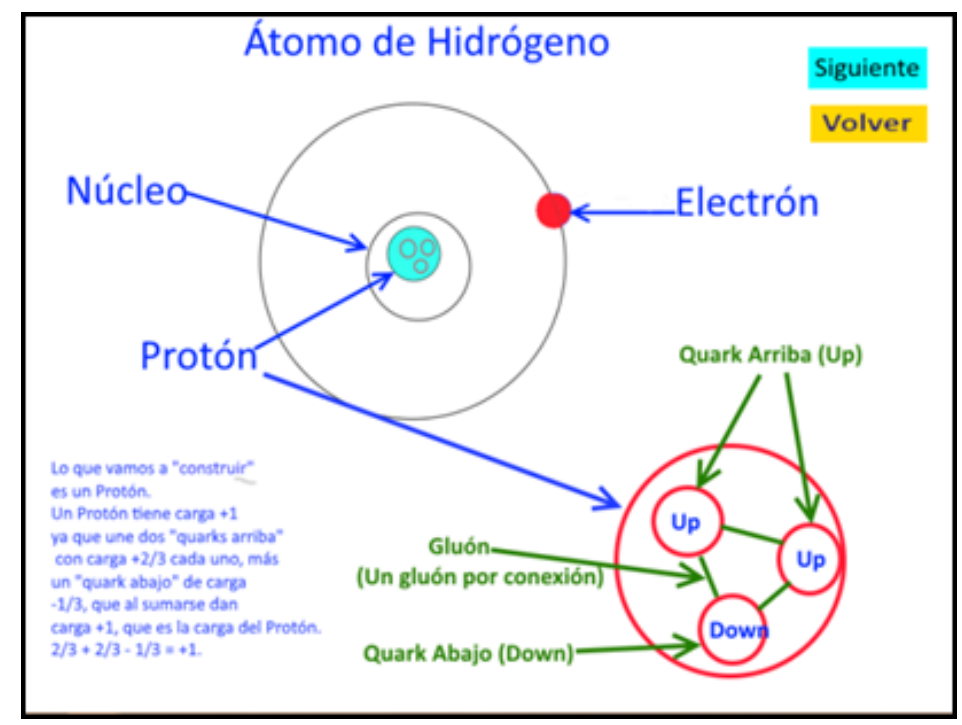

En la interfaz de práctica (Figura 2), el jugador tiene la posibilidad de conocer cuáles son los controles que le permitirán interactuar con el videojuego. De esta forma, puede aprender a controlar al personaje principal para cada nivel y etapa. El resto de las interfaces de juego son similares a esta.

Figura 2. Interfaz Práctica: Modo de Juego

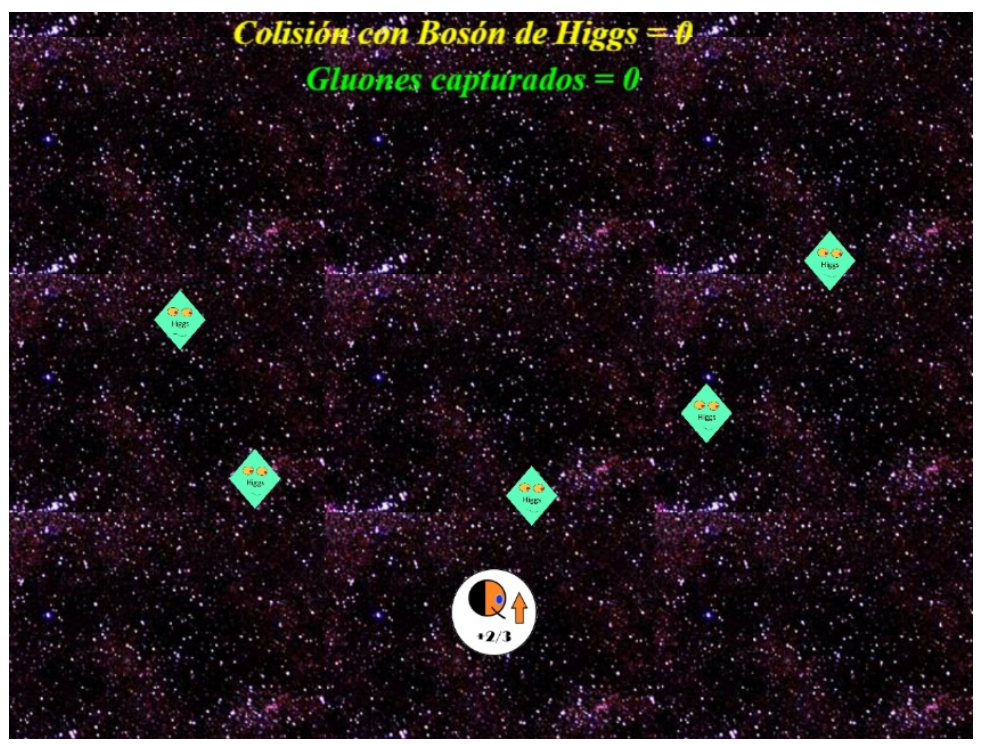

\section{Personajes}

Los personajes del videojuego forman parte de lo que se conoce como modelo estándar de la mecánica cuántica, del cual tomamos como personajes los que son necesarios para formar un átomo de hidrógeno, es decir, un quark arriba y un quark abajo que entre ambos forman un protón, el electrón, y finalmente los gluones y el bosón de Higgs. En el primer nivel el usuario es el quark arriba, y su objetivo será capturar los gluones que le ayudarán a formar el protón. En el segundo nivel el 
usuario es el gluón, y ahora debe capturar dos quarks arriba y un quark abajo para formar un protón. Finalmente, el jugador asume el control del protón, cuyo objetivo será capturar el electrón para formar el átomo de Hidrógeno. En los tres niveles, y en las diferentes etapas de cada uno el desafío fuera de cumplir el objetivo de captura es esquivar las interacciones con el bosón de Higgs, ya que sobre un cierto límite se pierde una vida.

Quark Arriba: personaje que debe capturar los gluones que serán los que le permitan unirse a otro Quark arriba y a un Quark abajo para formar un Protón. Su carga eléctrica es de $+2 / 3$ (más dos tercios). Pertenece al grupo de Leptones.

Quark Abajo: personaje que es capturado junto a dos Quark arriba para formar el protón. Su carga eléctrica es de $-1 / 3$ (menos un tercio). Pertenece al grupo de Leptones.

Gluón: este personaje es un Bosón, y es el que permite o es el "portador" de la interacción entre los quarks. No tienen carga eléctrica.

Bosón de Higgs: otro personaje Bosón, pero uno particular que es el encargado de "dotar" de masa a la materia. Este es el motivo que, si durante el juego se interactúa con muchos bosones de Higgs, las partículas desaparecen por su alto peso.

Electrón: partícula elemental que está presente en todos los átomos de la naturaleza. Su carga eléctrica es de -1 (menos 1). Pertenece al grupo de Leptones.

Protón: partícula formada por la interacción de tres quarks, dos quarks arriba y uno abajo, cuya suma de cargas da +1 (mas 1). Pertenece al grupo de Hadrones.

Figura 3. Personajes

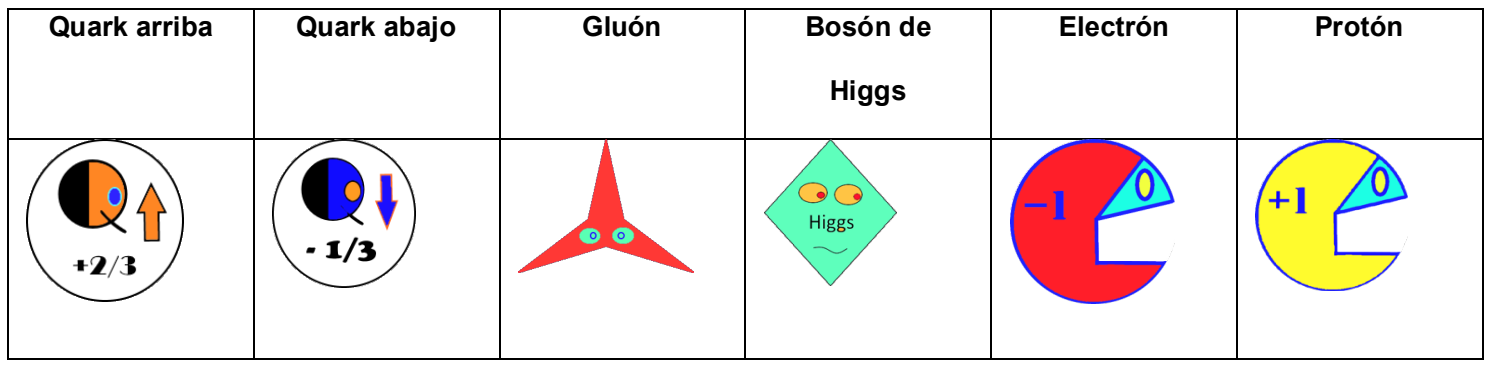

\section{Desarrollo}

El videojuego fue desarrollado en Gdevelop, un software de código abierto en su versión 4.0, utilizando su capacidad de poder compilar y publicar el videojuego final en plataforma web, con código HTLM5 (Rival, s.f.).

\section{Metodología}

\section{Usuarios}

Se trabajó con un curso de octavo año de enseñanza básica, compuesto por 14 niños 
y 23 niñas de una escuela particular subvencionada ubicada en la ciudad de Santiago de Chile. Los contenidos son pertinentes a la asignatura de Ciencias Naturales, por lo que la profesora a cargo de esta materia fue quien apoyó con el trabajo de los alumnos, y en particular con el grupo de que revisó los contenidos de manera tradicional.

La primera parte se trabajó con la totalidad de alumnos, mientras que en la segunda parte se hizo un trabajo separado en dos grupos, uno con enseñanza tradicional y otro que utilizó el videojuego.

\section{Instrumentos}

\section{Encuesta sobre el uso de videojuegos}

Este instrumento ha sido elaborado por los investigadores y busca encontrar el interés y relación de los niños con los videojuegos por medio de 13 preguntas. Cinco de estas preguntas consistían en una escala Likert con las opciones "nada", "un poco", "me da lo mismo”, “algo” y “mucho”. Estas preguntas fueron ¿Te gustan los videojuegos?, ¿Te gusta la música en los videojuegos?, ¿Te gustaría un videojuego que sirviera para estudiar?, ¿Te gustan los temas científicos? Y ¿Te gustaría ser parte de una investigación científica?

Las preguntas ¿En qué tipo de dispositivos juegas?, ¿Qué tipo de juegos prefieres?, ¿Prefieres jugar online, con amigos o solo? Y ¿Tienes conexión a Internet? contienen alternativas posibles de respuesta. Mientras que el resto de las preguntas (4) consistieron en respuestas abiertas: ¿Cuál/cuáles, son/es tu videojuego favorito?, ¿Cuánto tiempo juegas diariamente en horas?, ¿Cuál es tu personaje favorito? y ¿Puedes nombrar tus tres (3) asignaturas favoritas?

\section{Encuesta sobre elementos de Física Cuántica I}

Este instrumento fue desarrollado por los investigadores en conjunto con la profesora a cargo de la asignatura de Ciencias Naturales de 8vo básico. En esta encuesta, se solicita a los alumnos colocar el nombre de cada uno de los cuerpos de un átomo de hidrógeno presentadas en un esquema (Figura 4).

En este cuestionario había una segunda pregunta que tenía relación con conocer si sabían o habían escuchado hablar del Bosón de Higgs, los gluones, protones, electrones, neutrones y quarks. 
Figura 4. Partes del átomo de hidrógeno.

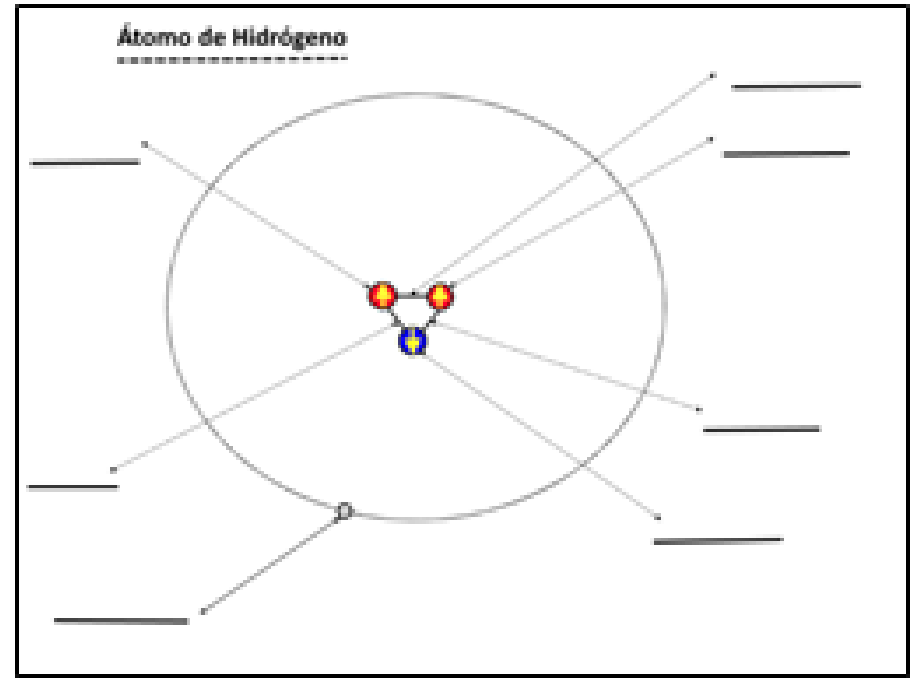

\section{Encuesta sobre elementos de Física Cuántica II}

En este tercer instrumento, elaborado por los investigadores y la profesora a cargo, se solicita a los niños, de una manera totalmente libre, completar las partes que conocen de un Átomo de Hidrógeno. Junto con esto, y también totalmente libre, se les pide dibujar un Átomo de Hidrógeno según los detalles que ellos quieran plasmar.

\section{Procedimiento}

El estudio se llevó a cabo en dos etapas. La primera etapa consistió en aplicar dos instrumentos. (A) El primer instrumento, "Encuesta sobre el uso de videojuegos", buscó conocer los intereses de los alumnos respecto a los videojuegos, su familiaridad con estos, con qué dispositivos era su acercamiento con los videojuegos y si tenían interés en conocer y jugar con videojuegos orientados al aprendizaje, entendiendo también cuáles eran sus asignaturas favoritas. (B) El segundo instrumento, "Encuesta sobre elementos de Física Cuántica I", se aplicó para saber el conocimiento nativo respecto del átomo de hidrógeno que tenían los alumnos.

La segunda etapa del estudio consistió en que la profesora de Ciencias Naturales les explicó a todos los alumnos cuáles eran las partes del átomo de hidrógeno, junto con entregarles una ficha elaborada con todo el contenido necesario para su revisión y aprendizaje. Durante las semanas que duró esta etapa, la profesora compartió con sus alumnos conocimiento acerca de los avances desde el mundo de la mecánica cuántica, relacionándolos con los temas que estaban estudiando.

Junto con el aprendizaje con la profesora, se seleccionó, según su interés, a un grupo de 9 niños ( 2 mujeres y 7 hombres) para utilizar el videojuego para ayudarlos a adquirir el conocimiento relacionado con las partes del átomo de hidrógeno. De esta manera, estos niños pudieron reforzar los elementos de la materia que contenía la ficha de contenidos de una manera lúdica. 
A partir del uso del videojuego, transcurrieron cinco días en que ambos grupos trabajaron con los contenidos asociados al átomo de hidrógeno, uno con la ficha y otro con el videojuego. Luego, sin previo aviso, se les aplicó un tercer instrumento, “Encuesta sobre elementos de Física Cuántica II", donde se les pidió nombrar las partes que componen un átomo de hidrógeno y hacer un esquema de este con un dibujo propio.

\section{Resultados}

Del instrumento "Encuesta sobre el uso de videojuegos" se conoce el perfil de los niños respecto al uso de videojuegos. Se obtiene que al 75,4\% de ellos les 'gustaba' o 'gustaba mucho' jugar ( $92 \%$ entre hombres y $64 \%$ entre mujeres), y que esto se hacía con diferentes 'dispositivos', siendo el más usado el teléfono inteligente (89\%), seguido con las consolas (51\%), luego el PC de escritorio (45,9\%) y finalmente el notebook (27\%). En cuanto al 'tiempo destinado a jugar', el promedio del curso es de 1 hora 45 minutos para los niños y 1 hora 24 minutos para las niñas. Dos niños declaran no jugar nada y los casos extremos son una niña que declara jugar 4 horas y media y un niño que declara jugar 5 horas. El 95\% de ellos tiene conexión a internet. Como resultado al instrumento "Encuesta sobre elementos de Física Cuántica I", era esperable que pudieran identificar las partes clásicas del átomo de hidrógeno, sin embargo, los resultados fueron muy diferentes, incluyendo además el quark arriba (quark up), el quark abajo (quark down) y los gluones. Los resultados de esta actividad se presentan en la tabla 1.

Tabla 1. Resultados actividad átomo de hidrógeno.

\begin{tabular}{|l|c|c|}
\hline \multicolumn{1}{|c|}{ Partícula } & \%Reconoce & \%No reconoce \\
\hline Gluón & 8.8 & 91.2 \\
\hline Quark Arriba (up) & 8.8 & 91.2 \\
\hline Quark Abajo (down) & 0 & 100 \\
\hline Electrón & 29.4 & 70.6 \\
\hline
\end{tabular}

El electrón logró un nivel de reconocimiento cercano al 30\% que de todas maneras es bajo respecto a lo esperado por ser una de las partes que se cree más conocida. Respecto a si conocían o al menos habían escuchado de los elementos del Bosón de Higgs, el electrón, el protón, el gluón, el neutrón y/o el quark, como era de esperar, el electrón, protón y neutrón son elementos reconocidos por los alumnos (Tabla 2).

Tabla 2. Resultados actividad de reconocimiento

\begin{tabular}{|l|c|c|}
\hline \multicolumn{1}{|c|}{ Partícula } & \%Reconoce & \%No reconoce \\
\hline Bosón de Higgs & 5.9 & 94.1 \\
\hline Electrón & 82.4 & 17.6 \\
\hline Protón & 58.8 & 41.2 \\
\hline Gluón & 11.8 & 88.2 \\
\hline Neutrón & 97.1 & 2.9 \\
\hline Quark & 2.9 & 97.1 \\
\hline
\end{tabular}

Revista Pensamiento Académico de la Universidad UNIACC Vol. $4 \mathrm{~N}^{\circ} 1,2021$ 
Los resultados para el segundo instrumento, "Encuesta sobre elementos de Física Cuántica II”, indican que al apoyar las materias de estudio con un videojuego se logra un mayor grado de asimilación por parte de los estudiantes.

Los niños que no tuvieron apoyo del videojuego nombraron en promedio 1,6 partes del átomo de hidrógeno, que sigue el mismo patrón antes de la actividad, reconociendo en general al protón y al electrón. Sin embargo, entre los niños y niñas que usaron el videojuego, el que menos nombró fueron 5 elementos, quedando el promedio en 6,4 partes (Figura 5).

En este mismo instrumento, donde debían dibujar átomo de hidrógeno con los detalles que recordara, la situación fue la misma, es decir, los niños que usaron el videojuego dibujaron en promedio 5,6 partes en promedio, contra 0,8 partes entre los niños y niñas que sólo recibieron la ficha informativa (Figura 6).

Figura 5. Promedio: Nombre partes que conozca de un átomo de hidrógeno.

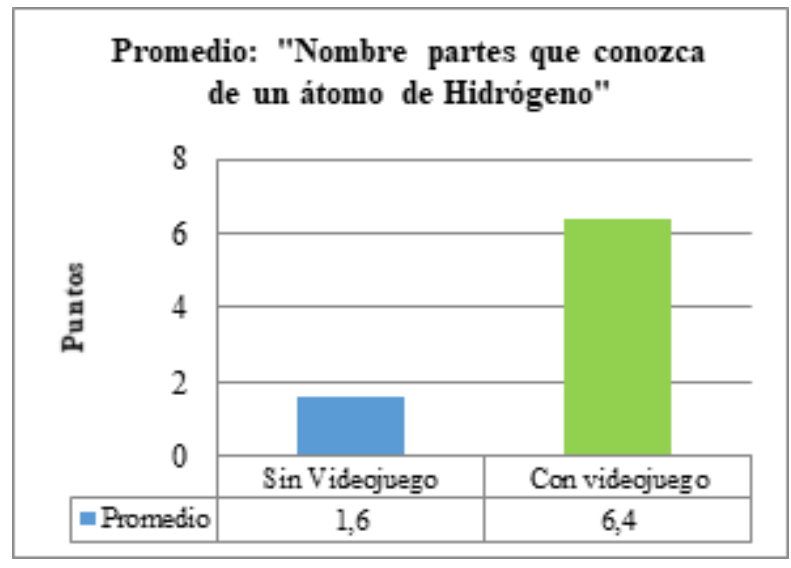

Figura 6. Promedio: Dibuja un átomo de hidrógeno con los detalles que conozcas.

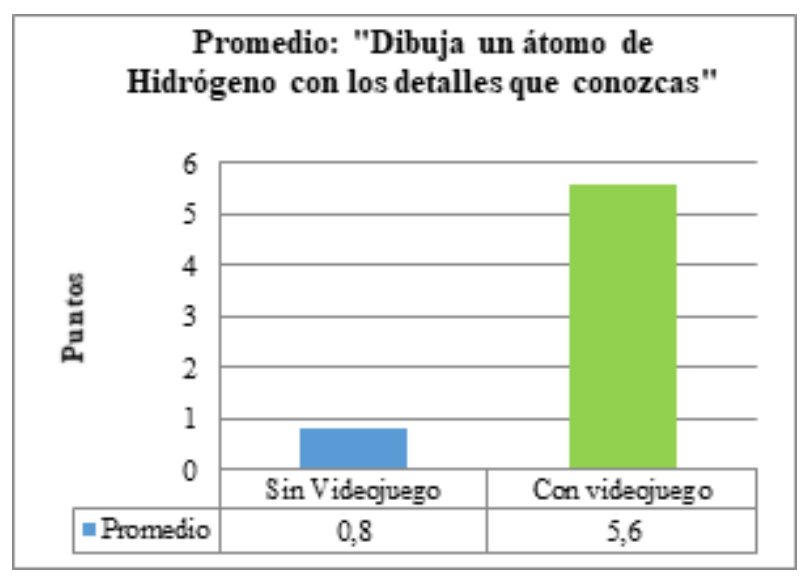

Además de la mejoría en los detalles presentados para quienes usaron los videojuegos, en la parte del dibujo hubo dos alumnos que utilizaron el esquema que entregaba el videojuego (Figura 7) y no el dibujo representado en la ficha. Creemos que esto es bastante notable desde el punto de vista de aprendizaje. 
Figura 7. Dibujos de átomo de hidrógeno.
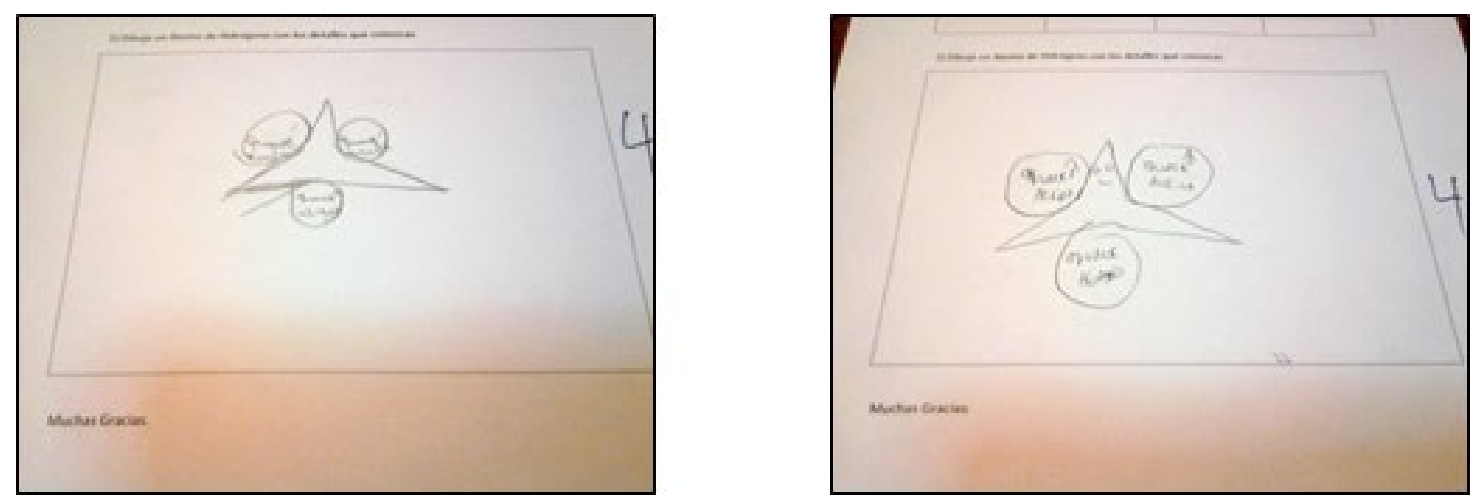

Una niña en particular, que no utilizó el videojuego, plasmó el dibujo del átomo de manera muy precisa, sin embargo, tanto el Bosón de Higgs como los quarks y gluones de todas maneras resultaron bastante desconocidos (Figura 8).

Figura 8. El mejor resultado para el esquema de átomo de hidrógeno.

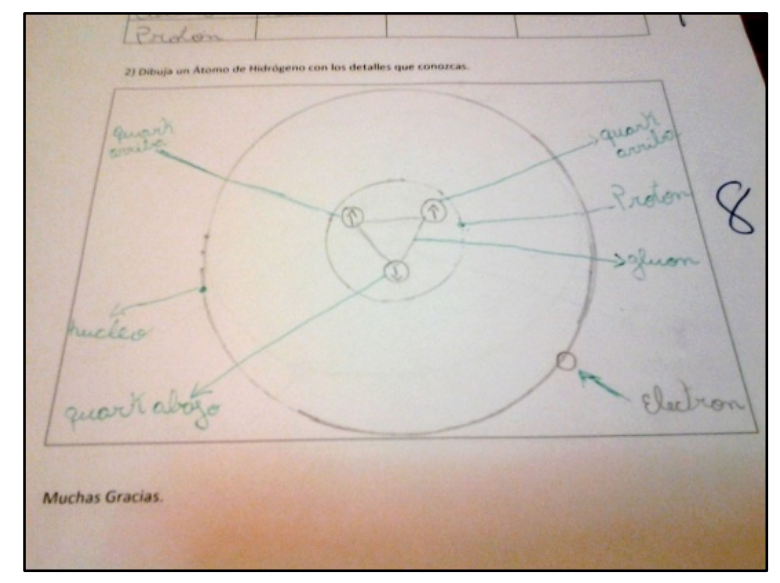

Considerando finalmente la sumatoria entre ambas preguntas, los que usaron el videojuego consiguieron 12 puntos en promedio contra 2,33 de los que no lo hicieron (Figura 9). Es importante destacar, que a nivel de las respuestas no hubo diferencias significativas entre niños y niñas.

Figura 9. Promedio: Encuestra sobre elementos de Física Cuántica II.

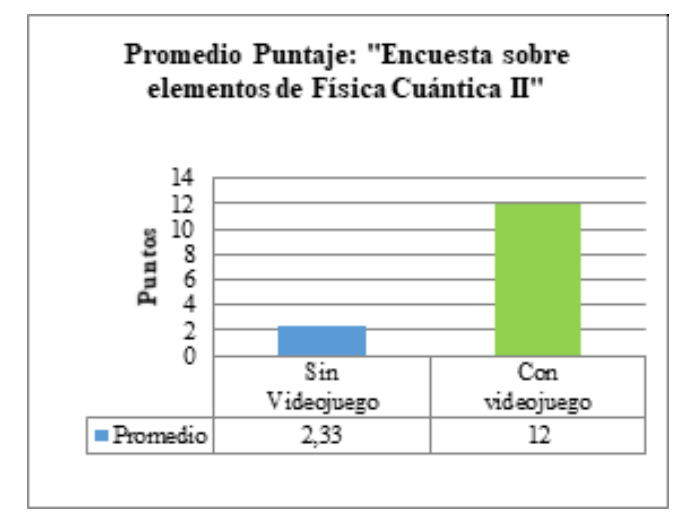




\section{Discusión}

A pesar del corto tiempo destinado al videojuego, lo que se puede apreciar con esta investigación, es que se tienen indicios ciertos de la ayuda que puede ser un videojuego como un factor altamente positivo para los estudiantes a familiarizarse con el aprendizaje del átomo de hidrógeno en un curso de octavo año de enseñanza básica en Chile. Tal vez se pudiera tener un sesgo en el modo de la selección del grupo de prueba, considerando que ellos manifestaron interés por ser parte de una investigación científica, lo que podría generar mayor entusiasmo que el videojuego mismo. Sin embargo, esto no debiera mermar el hecho de que el videojuego presenta un complemento importante en la diferenciación de los resultados expuestos.

Hoy en día, con todo el acceso a la tecnología que tienen los estudiantes, de manera cada vez más ubicua, los videojuegos se presentan como una muy buena alternativa para acercarlos a diferentes contenidos. Por tanto, resulta natural poder apoyarse en ellos para ayudar a los estudiantes a tener un contacto más natural con las materias de estudio.

Si se piensa en las casi dos horas diarias que muchos estudiantes destinan a los videojuegos, es genuino plantearse la pregunta de cómo es posible aprovechar dicha energía para los fines que el sistema de educación le exige al estudiante y que cada vez está más en línea con temas tecnológicos.

Es tanto el material escrito en este sentido, y en concordancia con los resultados aquí expuestos, que pensamos que no puede pasarse por alto el hecho de que hay que pensar en desarrollar videojuegos, que sin perder lo lúdico le permitan al estudiante mantener el foco en lo que persigue el profesor.

\section{Conclusiones}

Este videojuego se centra en el objetivo de mejorar el aprendizaje de las partes asociadas a un átomo de hidrógeno, considerando para ello el uso de un videojuego. En el ámbito del uso de tecnología para la educación, se les presenta el desafío de pensar en esta nueva herramienta, con el incentivo de crear instrumentos lúdicos que permitan tanto aprender como entretenerse.

Más allá de contar con una nueva experiencia donde se muestra lo poderosa que puede ser una herramienta como un videojuego, se abre también la posibilidad de un nuevo nicho de desarrollo que pueda incluir equipos dedicados a estas implementaciones, considerando diversos profesionales asociados a esta disciplina, en donde un rol primordial sería el de los expertos en contenidos.

Mientras tanto, los investigadores se embarcan en desarrollar un proyecto más amplio para lograr un set de videojuegos que comprenda mayor parte del programa de ciencias naturales de séptimo y octavo básico. Con esto, se podría profundizar el estudio del impacto de los videojuegos para la enseñanza de la ciencia, pudiendo ser una excelente herramienta para poner a disposición de los profesores del ámbito científico. 


\section{Agradecimientos}

A la profesora Ruth Fuentes Morales por su apoyo en esta investigación.

\section{Referencias}

Evaristo, I., Navarro, R., Vega, V. yNakano, T. (2016). Uso de un videojuego educativo como herramienta para aprender historia del Perú. RIED, Revista Iberoamericana de Educación a Distancia, 19(2), 35-52.

Farias, Y., Fornasier, M.S., Moro, L. y Morcela, A. (2016). Aprender energía a partir de un videojuego. $3^{\circ}$ Jornadas en Educación. Facultad de Humanidades, Universidad Nacional de Mar del Plata.

Galán, M. (2019, 14 de marzo). Ventajas de la Ludificación (Gamificación) en el Videojuego 'Manuelito en Búsqueda de la Atlantida'. Manuelito Investigador. http://manuelgalan.blogspot.com/2019/03/ventajas-de-la-ludificacion.html

Garrido, J. M. (2013). ¿Por qué los estudiantes juegan con videojuegos de estrategia?: algunos principios para la enseñanza. Revista Electrónica de Investigación Educativa, 15(1), 62-74. http://redie.uabc.mx/vol15no1/contenido-garridojm.html

González, C. (2014). Videojuegos para la Transformación Social. Aportaciones Conceptuales y Metodológicas [Tesis Doctoral, Universidad de Deusto]. Teseo. https://dialnet.unirioja.es/servlet/tesis?codigo $=118071$

James, J., Beaton, B., Csete, J. y Vogel, D. (2003). Mobile educational games. En: Lassner, D., McNaught, C. (eds.) Proceedings of ED-MEDIA, 801-802.

Lim, C., Nonis, D. y Hedberg, J. (2006). Gaming in 3D multi-user virtual students in Science lessons. British Journal of Educational Technology, 37(2), 211-231.

Ministerio de Educación. (2018). Programa de Estudio Octavo Básico, Ciencias Naturales. Gobierno de Chile. https://www.curriculumnacional.cl/614/articles20721_programa.pdf

Mora, A., Riera, D., González, C. y Arnedo-Moreno, J. (2015). A Literature Review of Gamification Design Frameworks. 7th International Conference on Games and Virtual Worlds for Serious Applications (VS-Games), 1-8. http://doi.org/10.1109/VSGAMES.2015.7295760

Ortiz-Colón, A., Jordán, J. \& Agredal, M. (2018). Gamificación en educación: una panorámica sobre el estado de la cuestión. Educação e Pesquisa, 44. https://www.scielo.br/j/ep/a/5JC89F5LfbgvtH5DJQQgHZS/:format=html\# 
PDLC2. (2015). Protectores de la Ciencia: Método Científico. //www.protectoresdelaciencia.cl/

Pellegrini, A., Blatchford, P. y Kentaro, B. (2004). A Short-term Longitudinal Study of Children's Playground Games in Primary School: Implications for Adjustment to School and Social Adjustment in the USA and the UK. Social Development 13(1), $107-123$.

Rival, F. (s.f.). Create your own games. GDevelop. https://gdevelop-app.com/

Sánchez, J. y Saenz, M. (2008). Resolución de problemas en ciencia a través de videojuegos móviles. Nuevas Ideas en Informática Educativa, 4. 15-22. http://www.tise.cl/volumen4/TISE2008/Documento03.pdf

Sánchez, J., Saenz, M., Ripoll, M. y Garrido, J. (2010). Usability of a Multimodal Videogame to Improve Navigation Skills for Blind Children. Transactions on Accesible Computing, TACCESS.

Sánchez, J., Saenz, M. \& Salinas, A. (2009). Videojuegos móviles para aprender y pensar en ciencias. En Vaz de Carvallho, C., Llamas, M. y Silveira, R. (Eds.), TICAI 2008: TICs para el Aprendizaje de la Ingeniería. (pp. 53-60) IEEE, Sociedad de Educación.

Science At Home. (2016). Games. https://www.scienceathome.org/games/

Sørensen, J., Pedersen, M., Munch, M., Haikka, P., Jensen, J.; Planke, T., Andreasen, M., Gajdacz, M., Mølmer, K., Lieberoth, A. y Sherson, J. (2016) Exploring the quantum speed limit with computer games. Nature, 532, 210-213.

Vargas, M. (2018, 1 de Octubre). La física y la matemática detrás del videojuego. Pesquisa Javeriana, Pontificia Universidad Javeriana Colombia. https://www.javeriana.edu.co/pesquisa/la-fisica-y-la-matematica-detras-delvideojuego/

Yip, S. (2015). Gamification: What place does it have in teaching and learning? Scan: The Journal for Educators, 34(4), 43-47. https://search.informit.org/doi/10.3316/informit.600110549538444 


\section{Mauricio Saenz Correa}

Ingeniero Civil en Computación y Magister en Ciencias, Mención Computación, Universidad de Chile; Diplomado en Habilidades Directivas, Universidad Adolfo Ibáñez; Egresado de Magister en Educación Superior, Universidad de Artes, Ciencias y Comunicación; Alumno en Diplomado de Experiencia De Usuario (UX DESIGN), Universidad Finis Terrae. Scrum Foundation Professional Certificate (SFPC). Profesor Asociado de la Escuela de Administración en la carrera de Ingeniería Informática Multimedia y en el Magister en Tecnología Educativa e Innovación de la Universidad de Artes, Ciencias y Comunicación. Gerente de Desarrollo y Operaciones (CTO) en Zweicom. Director en Interactio, Tecnologías para la Educación. Consultor en Tecnologías de la Información en Conexión Ingenieros. Evaluador del International Journal Elsevier Computers \& Education.

ORCID: 0000-0001-8406-9539

Email: msaenzc@gmail.com, mauricio.saenz@uniacc.edu

\section{Luis Pérez Hein}

Ingeniero en Informática Multimedia y Analista de Sistemas, Universidad de Artes, Ciencias y Comunicación; Diplomado en Filosofía de la Ciencia con mención en Física, y Diplomado en Filosofía de la Ciencias con mención en Sistemas Complejos, Instituto de Filosofía y Ciencias de la Complejidad. Director Técnico y Director de educación y Difusión del IFICC. Desarrollo Tecnológico del proyecto Fisicomics.cl.

Email: Iperezhein@yahoo.com 